\title{
A Framework for (Tele-) Monitoring of the Rehabilitation Progress in Stroke Patients
}

\section{eHealth 2015 Special Issue}

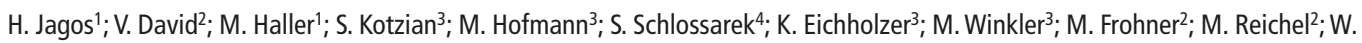
Mayr $^{1}$; D. Rafolt ${ }^{1}$

${ }^{1}$ Medical University of Vienna, Center for medical Physics and biomedical Engineering, Vienna, Austria; ${ }^{2}$ University of Applied Sciences Technikum Wien, Vienna, Austria; ${ }^{3}$ Neurological Rehabilitation Center Rosenhügel, Vienna, Austria; ${ }^{4}$ a 3 L e-Solutions $\mathrm{GmbH}$, Vienna, Austria

\section{Keywords}

Chronic disease, stroke, hemiplegia, gait, medical rehabilitation, motivation, exercise therapy, quality of life

\section{Summary}

Background: Preservation of mobility in conjunction with an independent life style is one of the major goals of rehabilitation after stroke.

Objectives: The Rehab@Home framework shall support the continuation of rehabilitation at home. Methods: The framework consists of instrumented insoles, connected wirelessly to a $3 \mathrm{G}$ ready tablet PC, a server, and a web-interface for medical experts. The rehabilitation progress is estimated via automated analysis of movement data from standardized assessment tests which are designed according to the needs of stroke patients and executed via the tablet PC application.

Results: The Rehab@Home framework's implementation is finished and ready for the field trial (at five patients' homes). Initial testing of the automated evaluation of the standardized mobility tests shows reproducible results.

Conclusions: Therefore it is assumed that the Rehab@Home framework is applicable as monitoring tool for the gait rehabilitation progress in stroke patients.

\section{Correspondence to:}

Harald Jagos

Medical University Vienna

Waehringer Guertel 18-20

AKH Wien/Ebene 4L

Email: harald.jagos@meduniwien.ac.at
Appl Clin Inform 2015; 6: 757-768

http://dx.doi.org/10.4338/ACl-2015-03-RA-0034

received: April 7, 2015

accepted: May 12, 2015

published: December 23, 2015

Citation: Jagos H, David V, Haller M, Kotzian S, Hofmann M, Schlossarek S, Eichholzer K, Winkler M, Frohner M, Reichel M, Mayr W, Rafolt D. A Framework for (tele-) monitoring of the rehabilitation progress in stroke patients. Appl Clin Inform 2015; 6: 757-768 http://dx.doi.org/10.4338/ACI-2015-03-RA-0034 


\section{Introduction}

Maintaining personal mobility is a key factor for the preservation of an independent and self-determined life style. Several types of chronic diseases, which are more prevalent with advanced age, can cause severe impairments in mobility, leading to a higher dependency, and a loss of the quality of life. One of the most common diseases, which leads to paralysis of the lower limbs, is stroke. During in-patient rehabilitation medical personnel conducts intensive therapeutic treatments for stroke patients [1]. After discharge from the in-patient setting into the domestic surrounding, patients often lose motivation to continue their treatment procedures, since they are not automatically provided with reminders by staff and advanced treatment options, like physical therapy, gait training, or gait assessment [2]. In the nationally funded project MISTRAAL a mobile movement analysis and feedback system for usage at home is being developed and tested. Stroke patients will be encouraged to monitor the progress in their therapy after the in-patient treatment. The rehabilitation progress is determined by the results of instrumented assessments and based on the development of improved movement quality. An instrumented shoe insole gathers movement parameters directly on the foot and delivers them to a health platform.

\section{Methods}

\subsection{System for Measurement and Monitoring Rehabilitation Progress}

A measurement system, embedded in a pair of orthopedic shoe insoles, in combination with a tablet PC as user interface, a server, and a web-interface for experts in the background constitutes the Rehab@Home framework ( $\triangleright$ Figure 1$)$.

The eSHOE measurement system contains state of the art sensors (3D Accelerometer, 3D Gyroscope, 3D Magnetometer, four pressure sensors) and electronics for data processing (PIC32, Bluetooth module, microSD card) ( Figure 2). In the following scenarios the measurement data is transmitted via Bluetooth 2.1 to the tablet PC. Data can be collected and transmitted at sample rates from 10 to $200 \mathrm{~Hz}$. eSHOE delivers raw data only, the actual basic signal processing (detection of crucial events) is taking place on the tablet-PC. More sophisticated methods, e.g. for detailed analysis of the data in order to provide therapists a closer insight, are applied offline, after the data have been stored on the server.

\subsection{Rehab@Home Concept}

In cooperation with sports scientists and physiotherapists from the Neurological Rehabilitation Center Rosenhügel in Vienna a small exercise program for the recovery of mobility for stroke patients was developed. The program was designed so that the exercises can be performed with and monitored by the instrumented insoles (eSHOE). A tablet PC serves as user interface where patients receive precise instructions and visual feedback and have the opportunity to consult a therapist (or technician) in case of a problem. The core of the exercise program is the collection of (standardized) mobility assessments. These are certain movement tasks that a person has to fulfil, mostly in a predefined timeframe. The time in which the subject is able to perform these tasks reflects his or her state of mobility. Two of these assessments shall be presented here. The timed "up and go" test (TUG) and the five times sit-to-stand test (STS5) ( $>$ Figure 3 and $>$ Figure 4 ).

The TUG test requires the subject/patient to initially sit on a chair. At a randomly occurring start signal the subject has to get up, walk three meters straight ahead (up to a mark), turn around, walk back to the chair, turn around again and sit down. The time for the completion of this task is recorded. There are several measures that indicate the state of mobility. A time over 14 seconds puts the subject at an increased risk of falling [3]. eSHOE can not only record the time needed to perform this test, but also collect movement data. These data allow for a more detailed quantitative analysis of the test, e.g. the segmentation into distinctive phases [4]. Using the tablet PC, in combination with a data server in the background, the patient is able to track the development of his/her test results over time in order to determine his/her rehabilitation progress. Through the expert interface thera- 
pists can access the more detailed information for the purpose of analyzing the measurement data in a scientific manner and in more depth.

The STS5 test was designed in order to determine functional lower limb muscle strength. The test subject is asked to sit on a chair and at a start-signal to stand up straight and sit down again, five times in a row, as fast as possible. Again, the time needed to complete this task is recorded $[5,6]$. The pressure data collected by eSHOE also allow a more detailed insight, e.g. whether the five repetitions were carried out rhythmically and whether the bodyweight is equally distributed on both legs.

Every exercise and assessment comes with a custom made instruction video that includes verbal directions via voice-over. Even though all users will receive a thorough introduction to the Rehab@Home program, the video gives them the chance to recap the details of the tests and exercises. Future plans envision a 1:1 relation for therapist and patient, which means that each patient has exactly one designated therapist who can access the control interface for his/her app settings or view his/her detailed measurements. Certain parameters, such as the level of difficulty for the assessments and tests, have to be adjusted individually to each patient by the medical experts.

In order to establish reproducibility, a group of healthy subjects $(n=6)$ was asked to perform these two assessments repeatedly over a period of three months as comparison. In addition, the application software for the tablet PCs was prepared. We present the preliminary results of both steps in the next section. In a clinical pilot study data from stroke patients $(n=30)$ were gathered during level walking for 90 seconds, TUG, and STS5. The final evaluation of the system will be a planned field trial, where five patients will receive the Rehab@Home system consisting of a pair of eSHOE instrumented insoles, a 3G-enabled tablet PC and an individually tailored exercise and assessment program.

\section{Results}

\subsection{Rehab@Home Tablet-PC Application}

The tablet PC app was designed and developed to be as easy to use as possible, taking into consideration the special needs of stroke patients. We assumed that most stroke patients (nowadays), due to their largely advanced age, are not that familiar with information technology. Additionally, due to their condition, they most likely suffer from sensory and locomotory impairments.

- Figure 5 shows the Rehab@Home app's home screen. The app also includes a help function, which can be accessed through a long click on the avatar ( Figure 6). The app's main functions are explained to the user through text as well as speech.

Furthermore the possibility to contact either a therapist or a technician was included, in case the user has a question or request ( Figure 7). In consultation with therapists pre-defined messages, from which the user can choose, are offered. That saves the user time (no need to type) and allows an early classification of the requests.

\subsection{Timed "up and go" Test}

The implementation of this assessment offers no live-feedback for the user, but it has, like all assessments and exercises, its own instruction video, which the user can watch as often as he/she wants or needs. When he/she performs this test for the first time, watching the video is mandatory. The user starts by putting on shoes with the instrumented insoles in place, selects the TUG test and presses "start" on the touch screen. After an appropriate delay to give him/her time to get in position for the test, he/she will hear a start command. Data transmission from eSHOE to the tablet PC starts simultaneously and the transmitted data is recorded on the tablet PC. The end-condition (user sitting down) is recognized automatically. With this event the test, along with the data recording is terminated. The user is presented with the test result in the form of time duration and a score. After the completion of the test, the detailed eSHOE data is transmitted to the central server, where it is available for the therapists via the expert web-interface.

The (visual) inspection shows that there are distinguishable patterns in the eSHOE data which offer the ability to evaluate the data automatically. Figure 8 shows two channels of the eSHOE 
data, a pictographic description of the TUG test (in top view), and the segmentation into six phases. In the graph with the eSHOE data, the $\mathrm{x}$-axis shows the time in seconds and the $\mathrm{y}$-axis acceleration in $\mathrm{m} / \mathrm{s}^{2}$ and angular velocity in degrees/s respectively. The upper eSHOE data stream in Figure 8 depicts the acceleration in anterior/posterior direction. Here the beginning of the forward movement, single steps (in straight direction!) and the end of the movement can be distinguished. When steps are performed in a more circular direction, e.g. when turning around, the anterior/posterior acceleration fails to deliver distinguishable data. But the gyroscope's longitudinal axis, otherwise limited in usefulness, can detect steps where the foot is turned. It even allows detecting how many steps it took to perform a turn, as can be observed in the second (lower) data stream in Figure 8 .

The first initial tests with healthy subject show mostly normally distributed results for the total duration ( 5 out of 6 ). However, for our six subjects greater than $50 \%$ of the results for the phases 1,3 , 5 and 6 have a normal distribution, but less than $50 \%$ of the results for the phases 2 and 4 are normally distributed. The level of significance was set to 0.1 . Figure 9 shows the distribution of the results for total time of the TUG test in the form of Box-Whisker-Plots for all subjects.

When patients (e.g. after a stroke) use the system to evaluate the status and progress of their mobility, they will achieve certain results which will be interpreted in relation to normative data and cut-off scores (e.g. 14 seconds for fall risk). The results will be presented to them in the form of a line chart, where they can monitor the progress of their performance ( Figure 12). The variation of their results as well as the detailed data (about the single phases) will be made available to therapists.

\subsection{Five Repetition Sit-to-Stand Test}

The data from the STS5 test can be analyzed in three different ways.

(1) Timing considering the time to stand up, sitting down, break times and times for the repetitions ( $>$ Figure 11).

(2) Load symmetry, which considers the distribution of weight between left and right side throughout the exercise execution ( $>$ Figure 10 ).

(3) Course of the center of pressure.

In the analysis of the test healthy subjects it was determined that five distinctive pressure peaks can be detected in the eSHOE data throughout the execution of the STS5 assessment.

The fact that it is possible to measure load distribution between the left and right foot enabled additional use of the STS5 test setting as a balance training exercise. The movement task for the patient is similar to the assessment (e.g. to get up five consecutive times from a chair) but the goal is to keep his/her weight distributed equally on both feet. For stroke patients with hemiplegia it is of great importance to regain the ability to put equal load on both feet. In $>$ Figure 10 the user interface with live load-feedback is displayed. Two bar charts represent the load on the left and the right foot respectively. As long as the patient keeps his weight within a certain range (e.g. $\geq 40 \%$ left and $\leq 60 \%$ right) the bar color remains green. When he/she leaves the centered region they change color, first to yellow then to red.

Results from the tests with healthy subjects show that it is not only possible to determine the time it took the subject to complete the test, but also to distinguish between the single movements during the test. Namely standing upright, with full load on the insoles, and sitting down with no load on the insoles.

\subsection{Progress Monitoring}

Since the patients' motivation can be increased by allowing them to monitor their rehabilitation progress [7], the results (according to the scoring system) over time are presented visually to the user. In order to relate to standard literature, a scoring system was implemented in the application which links the measurement/assessment results to easy-to-understand values in the form of a reward or achievement system. Figure 12 a) shows a screenshot as it is presented to the user directly after the completion of an exercise or assessment. He/she receives one to four stars (representing the test result) and is given the opportunity to rate the experience (good, neutral, bad) via 
clicking on an emoticon; b) shows the course of results over a period of time, which gives the user an overview of the development of his/her performance at a specific test or exercise over time.

\section{Discussion}

After the successful integration of eSHOE hardware, tablet PC (plus application software), and server an initial proof of concept for the implementation of a Rehab@Home system was completed. The concept for the rehabilitation support program as well as the (design of the) screens of the Rehab@Home app were optimized in close cooperation and in several iterations with the experts from the neurological rehabilitation center Rosenhügel. Subsequently, the app's usability will be tested for acceptance and adequacy. Furthermore a feasibility study will be carried out, where five patients will test the complete system at their homes for one month after discharge from the rehabilitation center. In the meantime the signal processing algorithms for the automated evaluation of assessments and exercises will be optimized and improved. Hence, we anticipate that the results of the distinctive phases of the TUG test will also approach normal distribution, along with the total duration. The signal processing algorithms need to be adapted to pathological gait parameters, in our case to gait parameters of stroke patients. Based on the preliminary results, the Rehab@Home framework looks promising for a future use as (tele-) monitoring tool.

Since continuous data transmission from eSHOE to the tablet-PC is of the essence in all the assessment scenarios and signal processing, for the time being is performed on the tablet-PC, Bluetooth 4.0 (Low Energy) was no option. It requires data to be transmitted "packet-wise" (small amounts of data at large time intervals) and at low data rates $(<22 \mathrm{kBit} / \mathrm{s})$.

\section{Clinical relevance:}

eSHOE proves to be a valuable supplement to intramural, instrumented gait analysis. It is conceivable to integrate eSHOE into the clinical practice in the future, instrumenting and supporting the analysis of mobility assessments. Furthermore eSHOE provides the possibility to perform measurements/assessments in everyday life situations and locations [8].

\section{Conflict of interest:}

The authors declare that they have no conflicts of interest in the research.

\section{Human Subjects Research:}

All procedures involving patients have been reviewed in compliance with ethical standards of the responsible committee on human experimentation. The pilot study received a positive vote form the local ethics committee of the municipality of Vienna (EK 14-050-0314). 


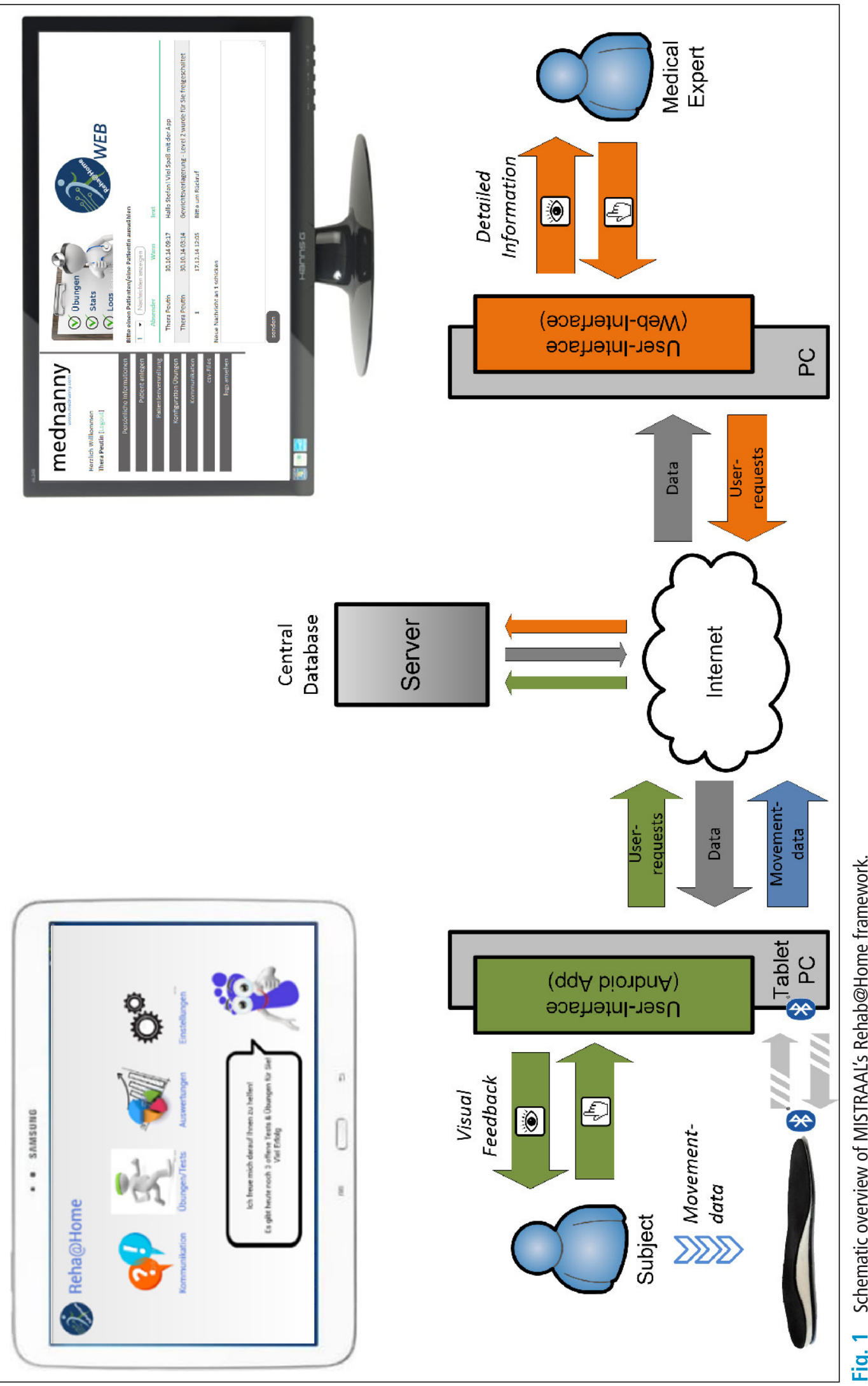




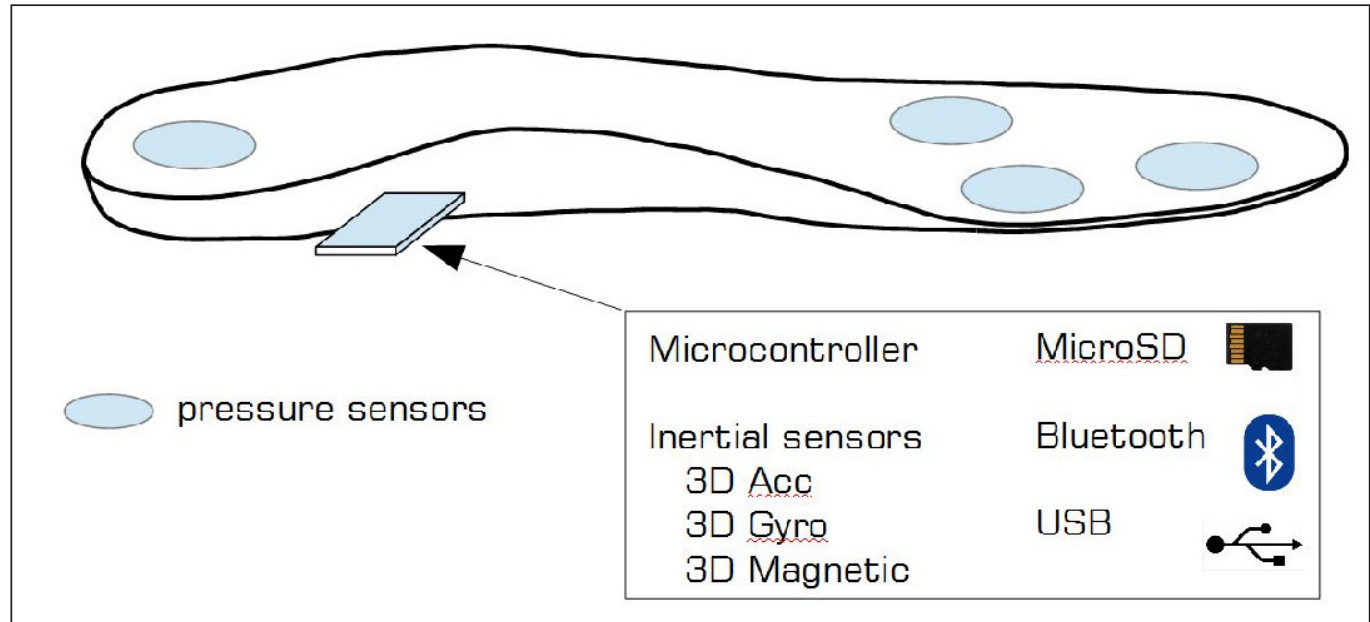

Fig. 2 Schematic diagram of the eSHOE system with all its components.

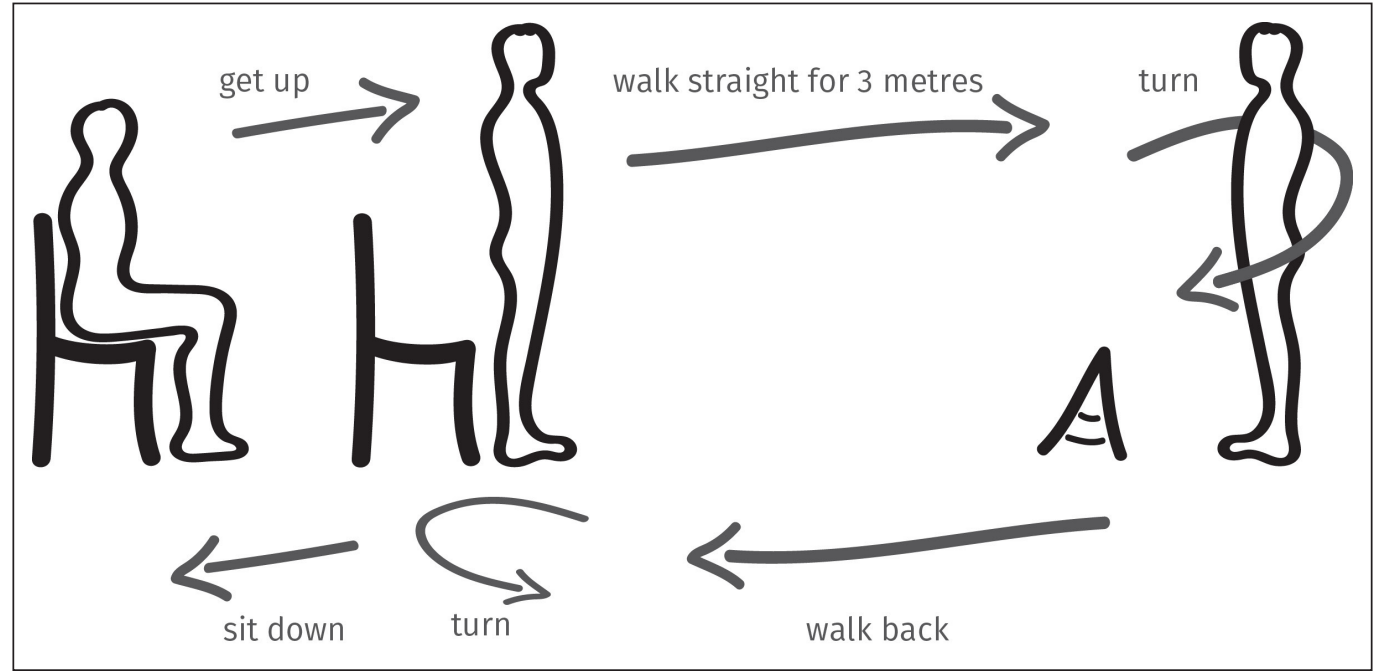

Fig. 3 The timed "up and go" test.

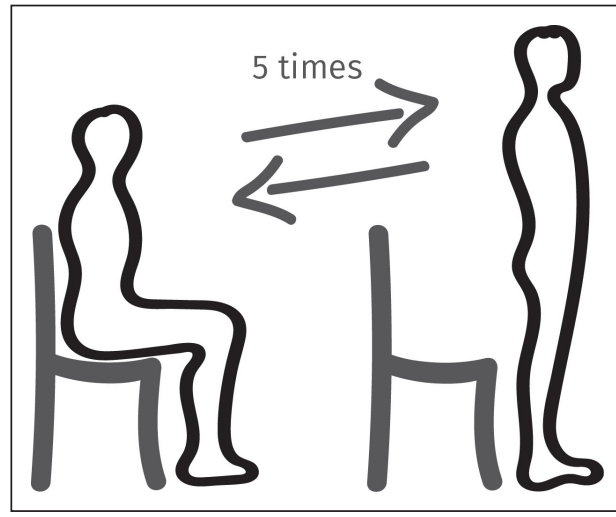

Fig. 4 The five times sit-to-stand test or chair rising test. 


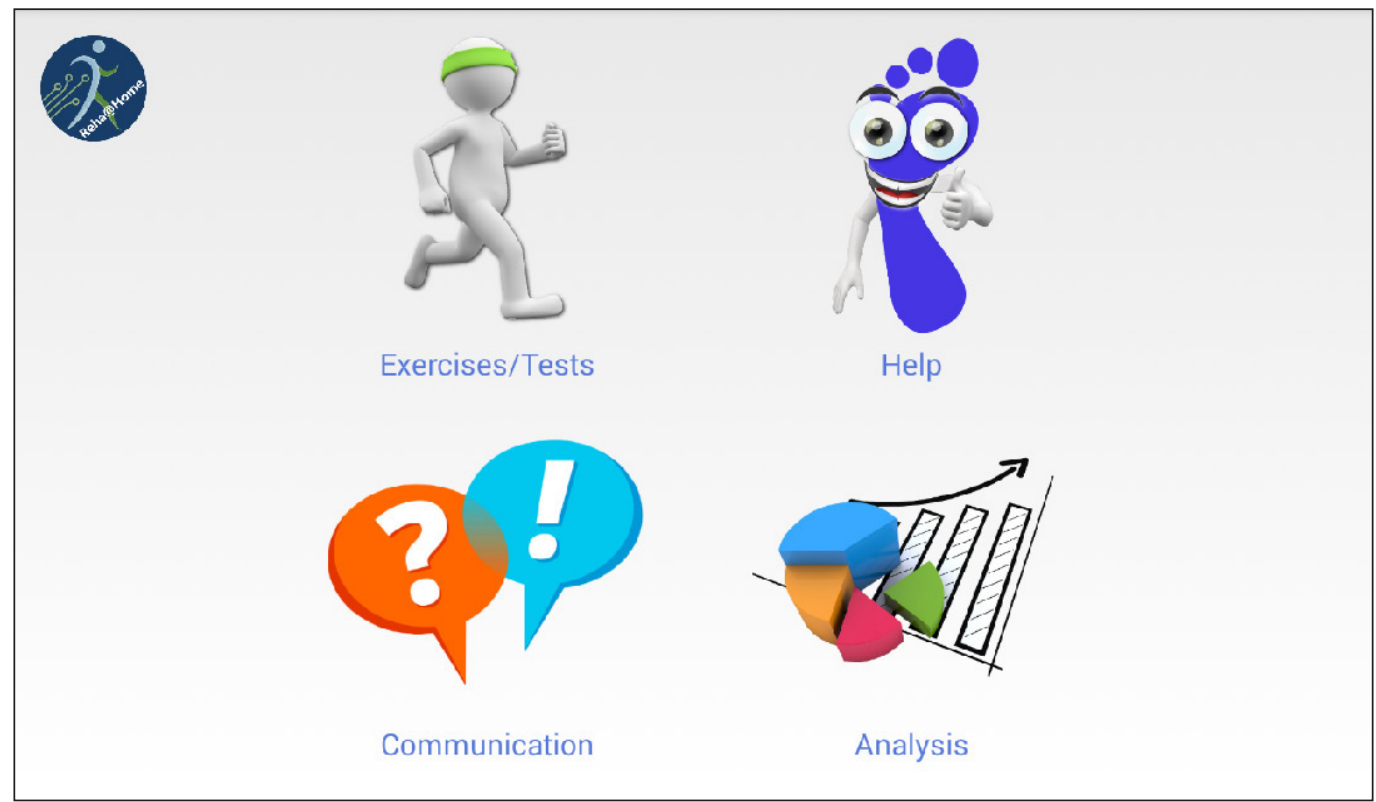

Fig. 5 MISTRAAL's Rehab@Home app

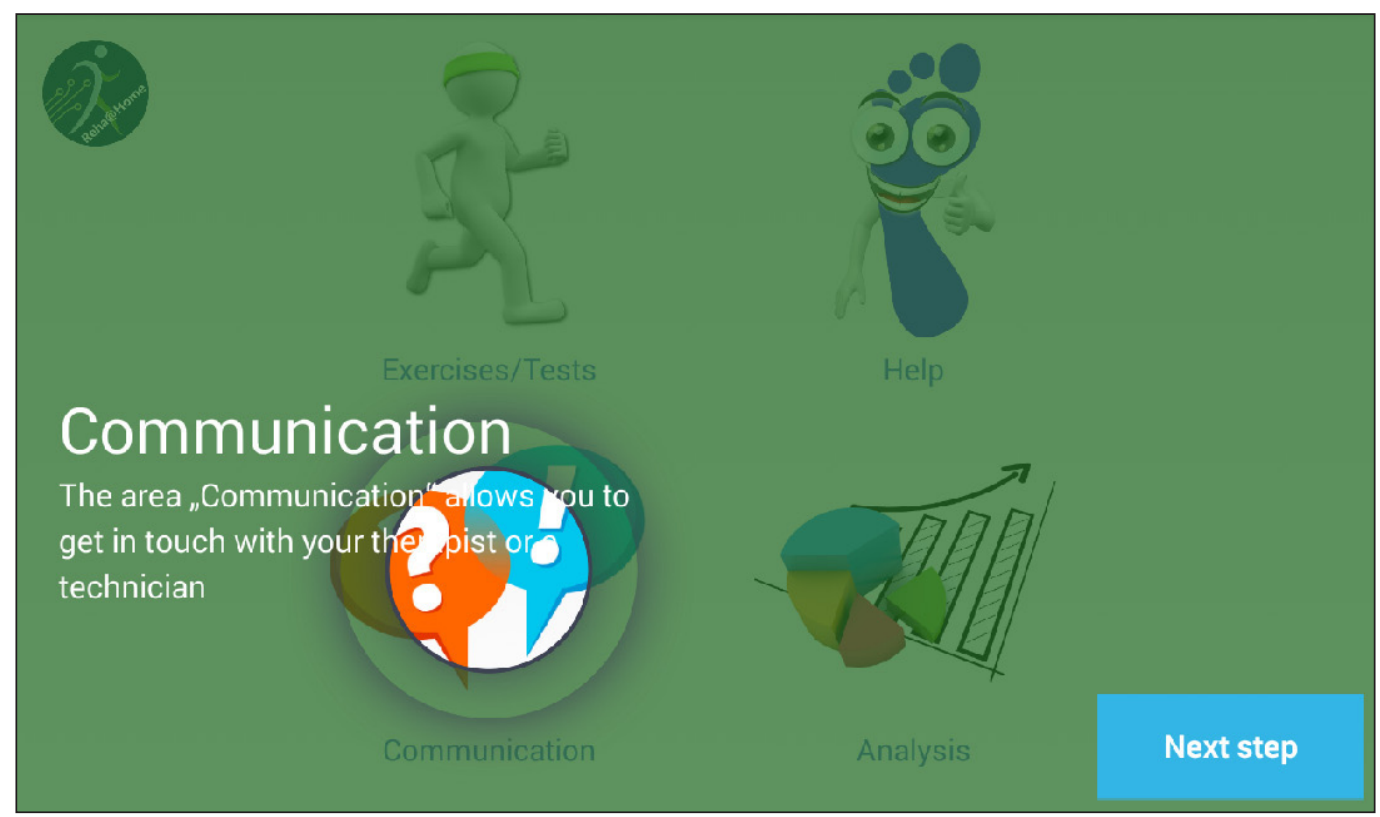

Fig. 6 The Rehab@Home app's help function, executed on the home screen. 


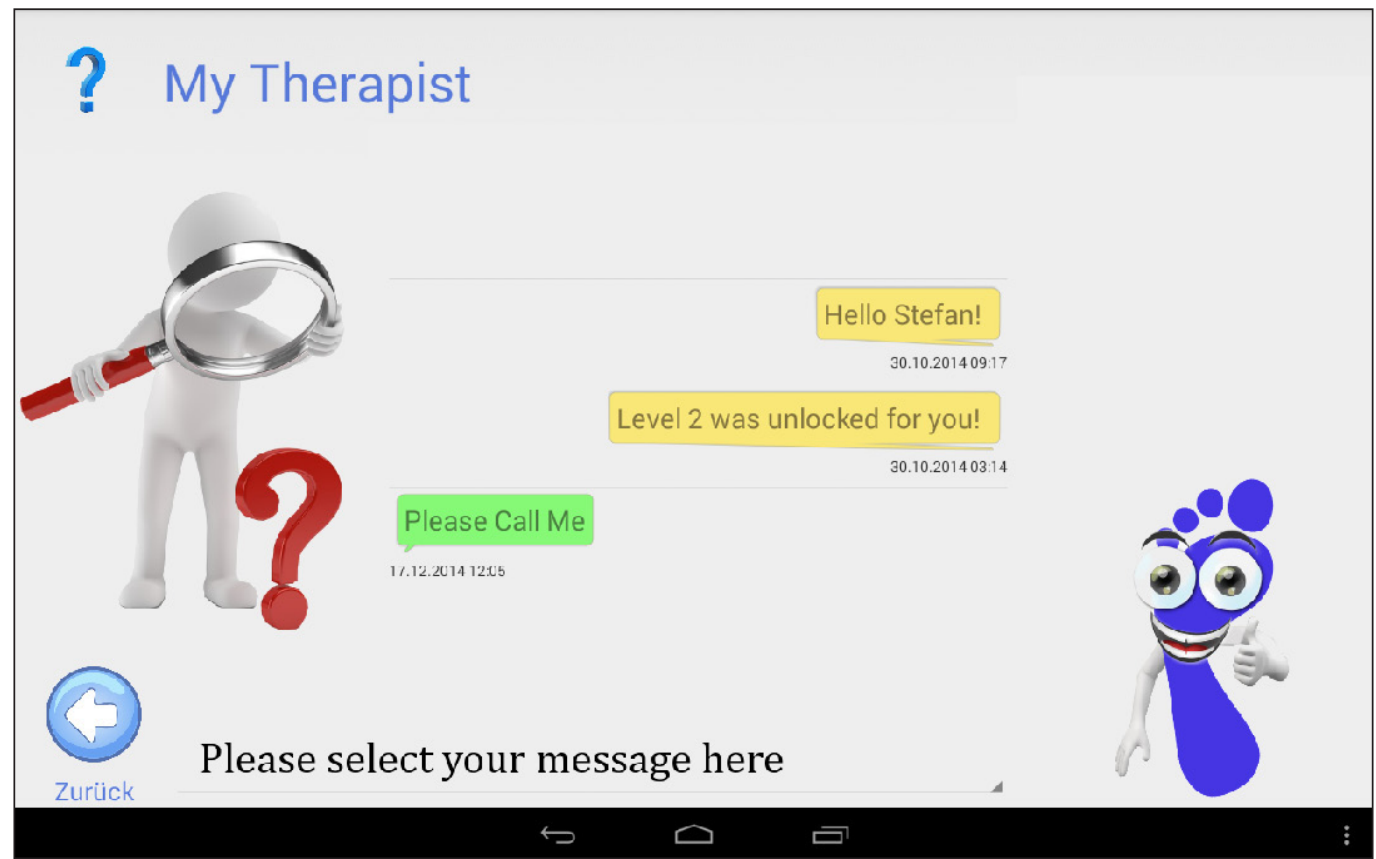

Fig. 7 The Rehab@Home app's communication interface. Here patients can select from a variety of pre-defined messages or hand-type their own message. The app contains two of these interfaces, one to contact a therapist and another to get in touch with a technician.

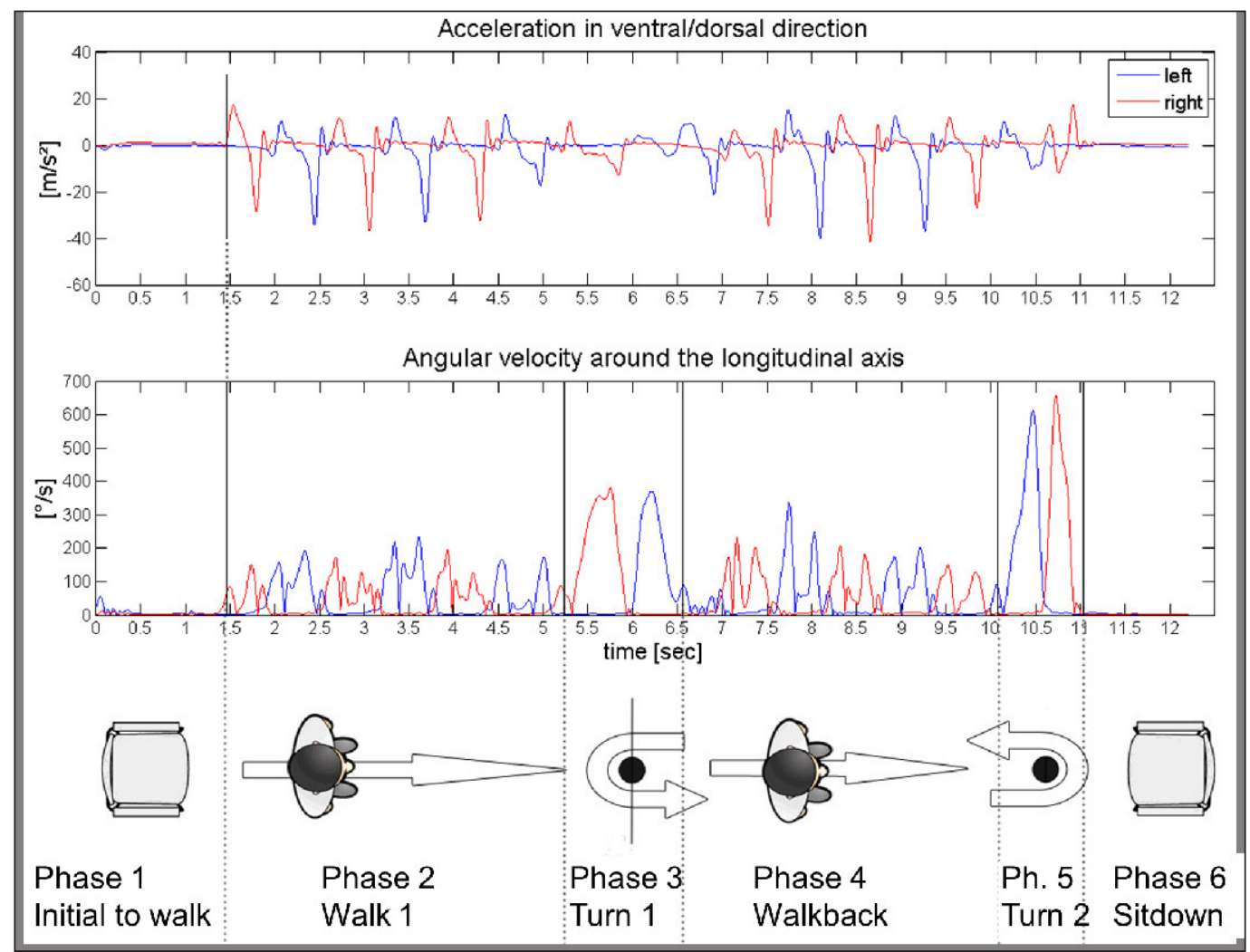

Fig. 8 eSHOE data from the TUG test. Upper: acceleration in anterior/posterior direction. Middle: angular velocity around the longitudinal axis. Lower: schematic representation of TUG test procedure. 


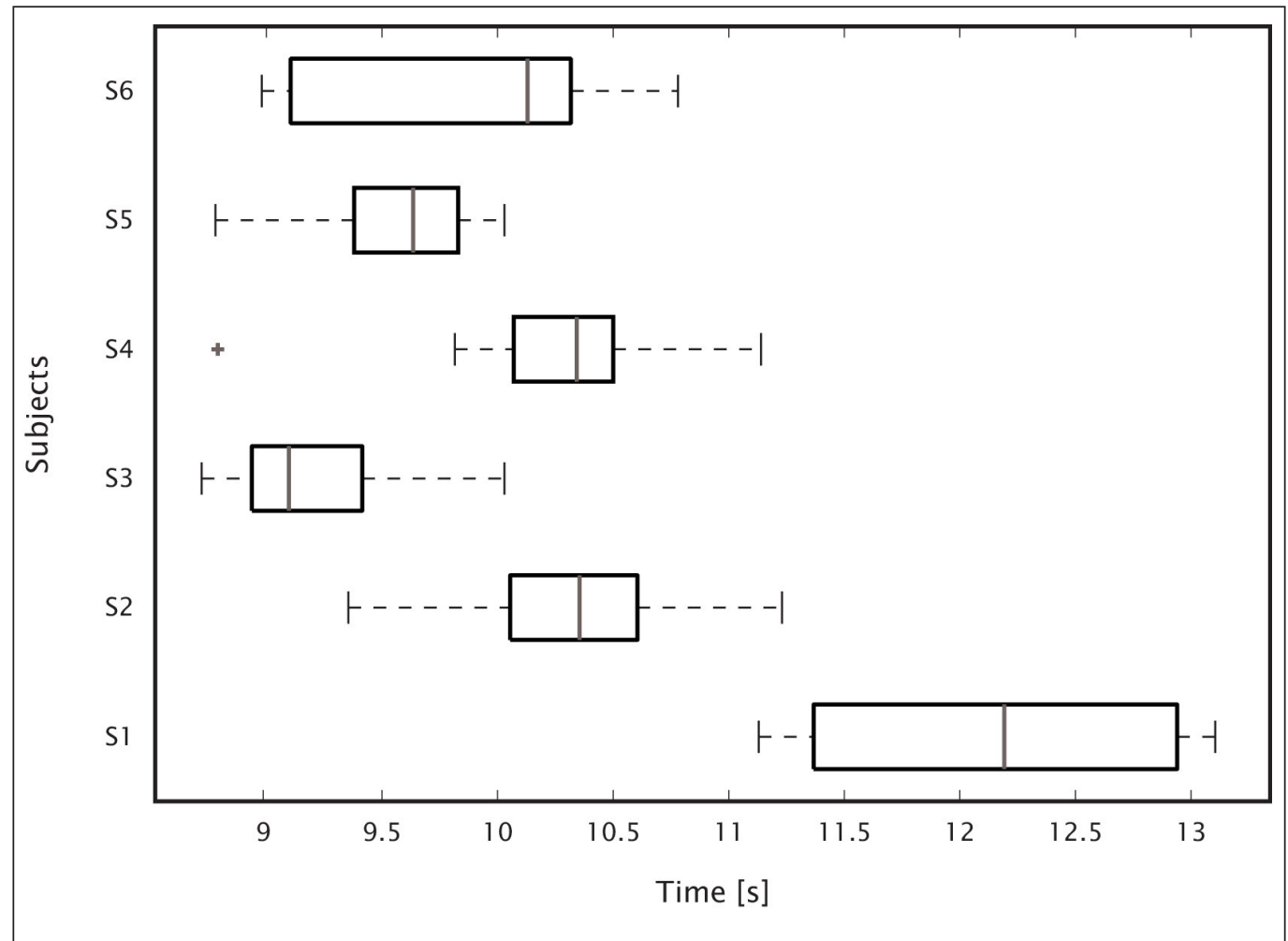

Fig. 9 Box-Whisker-Plots of the six healthy subjects' TUG test results. $x$ - and $y$-axis show the time in seconds and the subjects results for total duration of the TUG test, grouped by subjects (S1 to S9), respectively. Whisker-length: Q1-1.5 x (Q3-Q1) and Q3+1.5 x (Q3-Q1).

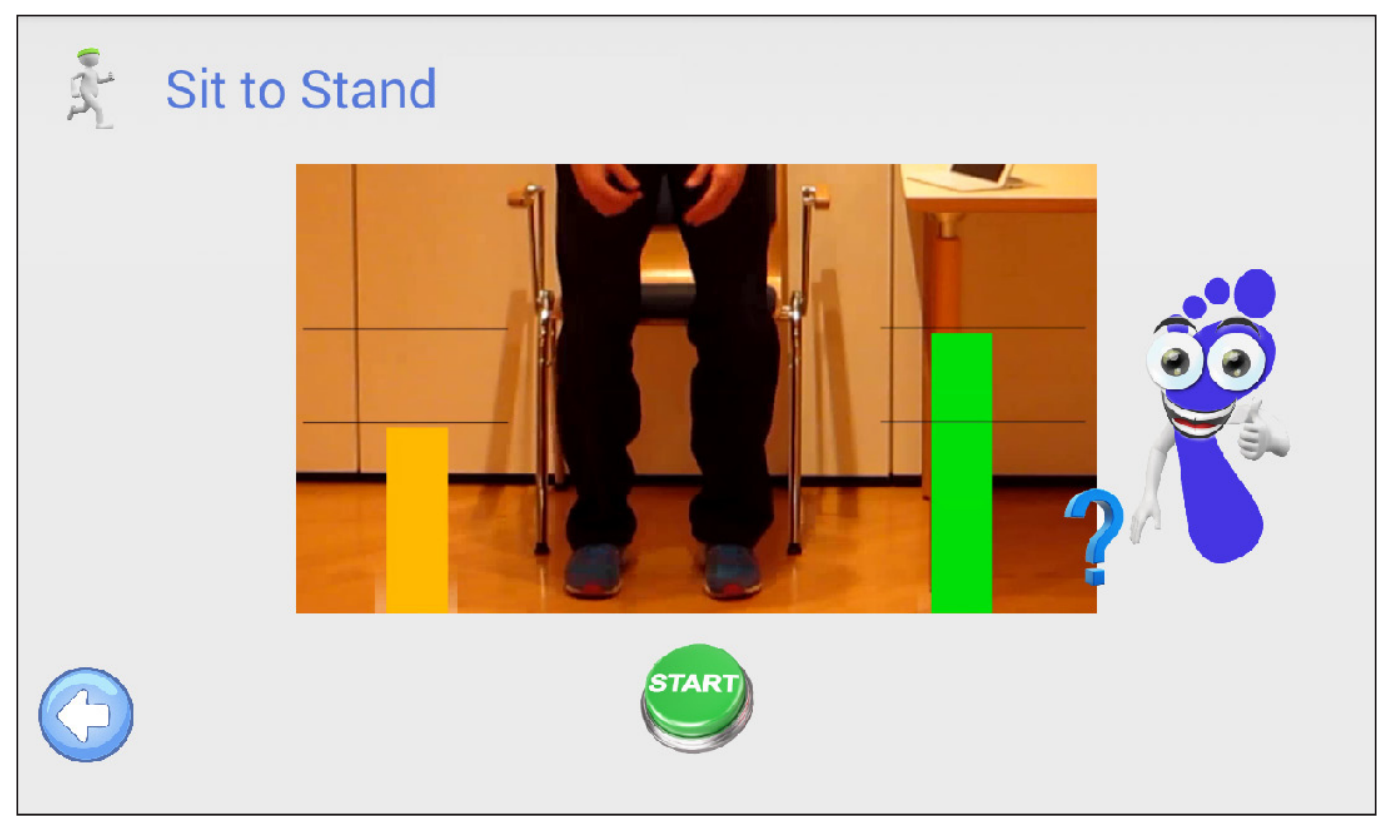

Fig. 10 User interface for the five repetition sit-to stand test (STS5). Showing a still photograph of a person standing up from a chair and two bar charts, representing the sum of pressure under each foot. The horizontal lines indicate a range of pressure distribution which means equal pressure distribution on both feet. 


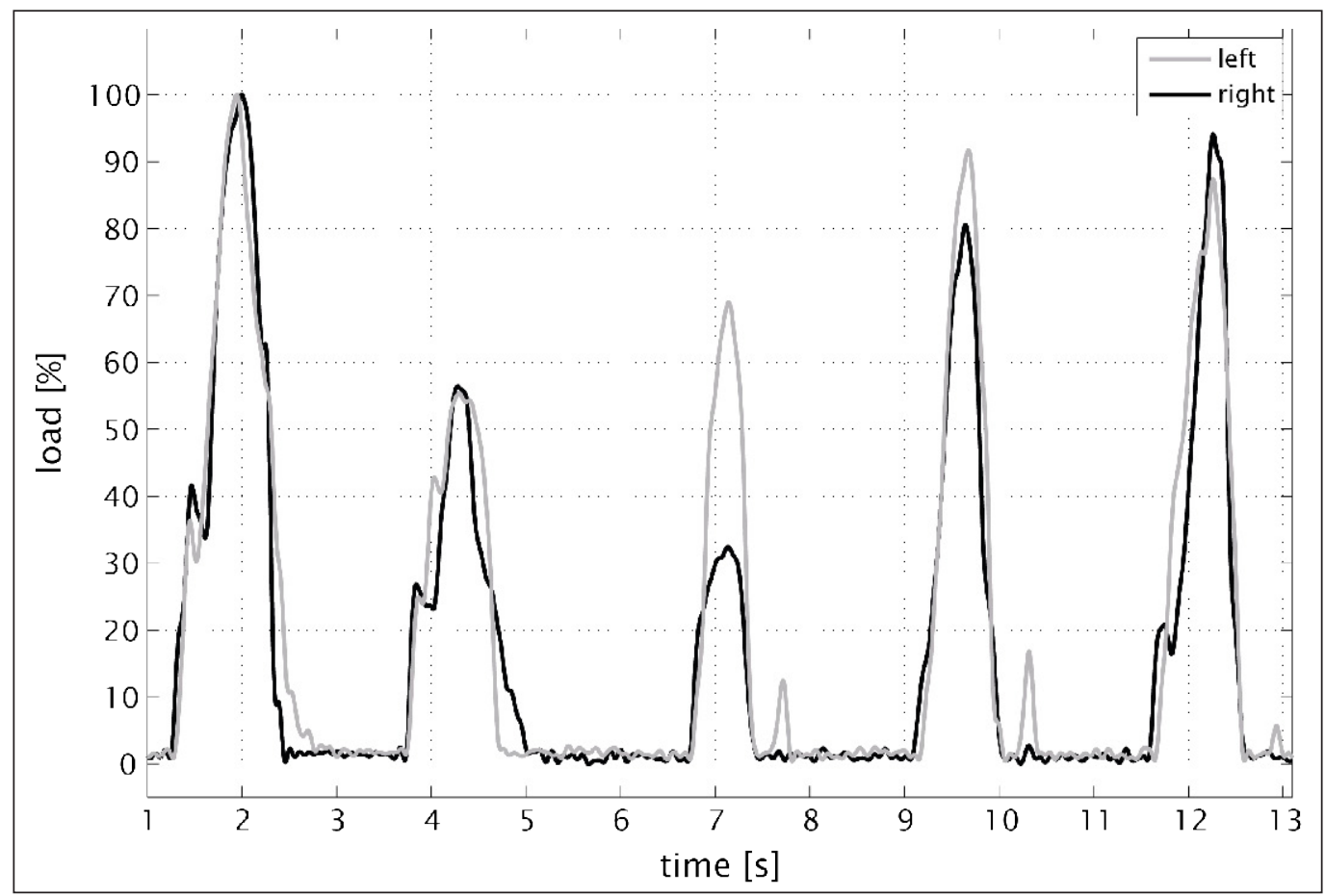

Fig. 11 eSHOE raw data, collected during a STS5 test. Plotted here are pressure sums from the left and the right foot over time.

\section{Analysis}
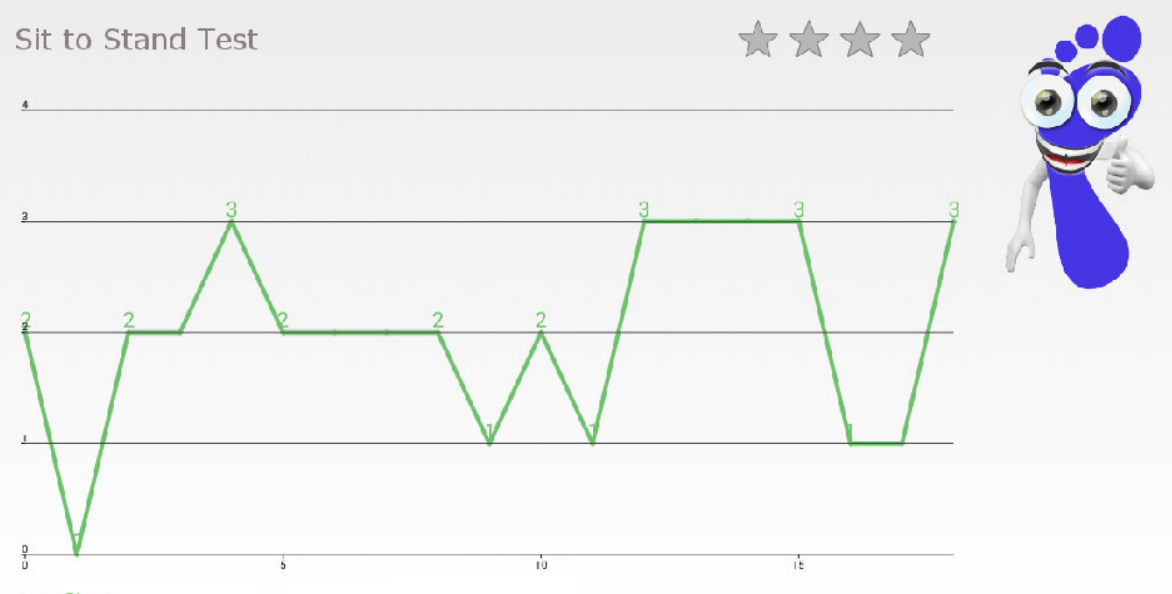

$$
\text { - Stars }
$$

Fig. 12 Display of an overview of all results that occurred in one test (five times sit-to-stand test) over a certain period of time. The upper line represents the time duration and lower line the achieved scores (one to four stars). 


\section{References}

1. Duncan PW, Zorowitz R, Bates B, Choi JY, Glasberg JJ, Graham GD, Katz RC, Lamberty K, Reker D. Management of Adult Stroke Rehabilitation Care: A Clinical Practice Guideline. Stroke 2005; 36(9): e100-e143.

2. Jørgensen HS, Nakayama H, Raaschou HO, Olsen TS. Recovery of walking function in stroke patients: The copenhagen stroke study. Archives of Physical Medicine and Rehabilitation 1995; 76(1): 27-32.

3. Shumway-Cook A, Brauer S, Woollacott, M. Predicting the probability for falls in community-dwelling older adults using the Timed Up \& Go Test. Physical therapy 2000; 80(9); 896-903.

4. Oberzaucher J. iAssessment - Aspekte eines instrumentierten Sturzrisiko-assessments basierend auf einer extramuralen Gang- und Bewegungs-analyse - im Hinblick auf eine Anwendung im Bereich des Ambient Assisted Living [dissertation]. Vienna (Austria): Vienna University of Technology 2011.

5. Beninato M, Portney LG, Sullivan PE. Using the International Classification of Functioning, Disability and Health as a framework to examine the association between falls and clinical assessment tools in people with stroke. Phys Ther 2009; 89(8): 816-825.

6. Bohannon RW. Reference values for the five-repetition sit-to-stand test: a descriptive meta-analysis of data from elders. Percept Mot Skills 2006; 103(1): 215-222.

7. Popović MD, Kostić MD, Rodić SZ, Konstantinović LM. Feedback-mediated upper extremities exercise: increasing patient motivation in poststroke rehabilitation. Biomed Res Int 2014; 2014: 520374.

8. Pils K, Jagos H, Wassermann C, Chhatwal C. Evaluierung einer mobilen Ganganalyse - eSHOE. Phys Med Rehab Kuror 2014; 24: 1-14. 